\title{
Interleukin-6 in the Maternal Circulation Reaches the Rat Fetus in Mid-gestation
}

\author{
JOVANNA DAHLGREN, ANNE-MAJ SAMUELSSON, THOMAS JANSSON, AND AGNETA HOLMÄNG
}

\begin{abstract}
Göteborg Pediatric Growth Research Center [J.D.], Institute for the Health of Women and Children, Queen Silvia Childrens Hospital, 41685 Göteborg, Sweden; Wallenberg Laboratory [A.-M.S., A.H.], Cardiovascular Institute, The Sahlgrenska Academy at Göteborg University, 41345 Göteborg, Sweden; Institute of Physiology and Pharmacology [T.J.], The Sahlgrenska Academy at Göteborg University, 40530 Göteborg, Sweden; Department of Obstetrics and Gynecology [T.J.], University of Cincinnati, Cincinnati, OH 45267
\end{abstract}

\begin{abstract}
Maternal systemic infection during pregnancy may expose the fetus to infectious agents and high levels of mediators of the resulting inflammatory response, such as IL-6 (IL-6). Increased fetal and maternal levels of IL-6 have been associated with adverse neonatal outcome but might also stress the fetus and contribute to cardiovascular and neuroendocrine dysfunction in adulthood. It is unclear whether interleukines cross the placental barrier, although this matter has been little studied. The aim of this study was therefore to investigate if IL-6 administered to pregnant rats in vivo is transferred to the fetus. We injected ${ }^{125}$ I IL- 6 i.v. to pregnant dams at gestation day 11-13 (mid-gestation) or 17-19 (late gestation). We found ${ }^{125}$ I-IL-6 in the exposed fetuses as well as in amniotic fluids. Fetal ${ }^{125}$ I-IL-6 levels were markedly higher in animals injected in mid-gestation compared with late pregnancy $(p<0.01)$. This difference was mirrored in a 15 -fold higher unidirectional materno-fetal clearance for ${ }^{125}$ I-IL-6 in mid-gestation $(p<0.01)$. We conclude that the permeability of the rat placental barrier to IL-6 is much higher in mid-gestation than in late pregnancy. Maternally derived IL-6 may directly induce fetal injury but also stimulate the release of fetal stress hormones resulting in stimuli or insults in neuroendocrine structures and hormonal axes which might lead to disease at adult age. (Pediatr Res 60: 147-151, 2006)
\end{abstract}

$\mathrm{M}$ aternal infections such as pneumonia and urinary tract infection are well known to stimulate immune cells to produce cytokines such IL-6 $(1,2)$. IL-6 is one of several cytokines linked to preterm labour associated with infection $(3,4)$ but also in the absence of apparent clinical infection (5). The intra-amniotic inflammatory response has also been associated with white matter injury and cerebral palsy and IL-6 has been shown to be an independent predictor of these serious disorders $(6,7)$.

Several diseases in adults, including hypertension, coronary heart disease, stroke, obesity, and type 2 diabetes may have

Received August 9, 2005; accepted March 18, 2006

Correspondence: Jovanna Dahlgren, Ph.D., Department of Pediatrics/GP-GRC, The Queen Silvia Children's Hospital, Institute for the Health of Women and Children, The Sahlgrenska Academy at Göteborg University, SE-416 85 Goteborg; jovanna. dahlgren@vgregion.se

This work was supported by grants from the Wilhelm and Martina Lundgren's Foundation, the Tore Nilsson Foundation, the Swedish Medical Society, the Göteborg Medical Society, and the Swedish Heart Lung Foundation. The authors are grateful to G Ekeroth for statistical support.

DOI: $10.1203 / 01 . p d r .0000230026 .74139 .18$ their origins in suboptimal intrauterine environment (8). The proposed underlying mechanism is early programming, a process whereby stimuli or insults in early life have long-term effects on the individual's structure, physiology, metabolism, and mental functions in later life. Prenatal stresses such as infection, inflammation and increased exposure to glucocorticoids adversely affect the developing fetus $(9,10)$. In addition, prenatal exposure to IL-6, early and late in pregnancy, appears to cause hypertension, insulin resistance, elevated stress response, with diminished feed-back control and dysregulation of the hypothalamic-pituitary-adrenal axis activity during adulthood $(11,12)$. Thus, immune cytokines seem to play a vital role in the programming of neuroendocrine functions during ontogenesis.

While it is known that the placenta may be permeable to cytokines if damaged, little is known about the permeability for IL-6 in a healthy placenta (13). IL-8 is not transferred across the term human placenta perfused in vitro (14). In contrast, Zaretsky et al. using similar techniques recently reported transfer of IL- 6 across the human term placenta in both the maternal-fetal and fetal-maternal directions (15). Moreover, some studies show that the placenta appears to be permeable to infective agents in early pregnancy $(16,17)$. Low amounts of IL-1 $\beta$ (IL-1 $\beta$ ), IL- 6 and tumor necrosis factor- $\alpha$ (TNF- $\alpha$ ) have been found to cross intact human amnion, chorion and deciduas in culture (18).

Previous studies in pregnant rats have demonstrated that maternal administration of IL-6 results in programming effects on adult offspring, but it is not clear if this is mediated through stimulation of the maternal HPA axis or by direct fetal exposure to IL-6 $(11,12)$. It has been hypothesized that maternally generated cytokines cross the placenta, e.g., during maternal systemic inflammation response, and enter the fetal circulation (19). The permeability characteristics of the rat placenta have been studied in some detail $(20,21)$, however, to the best of our knowledge, whether significant transplacental transfer of IL-6 occurs in this species has not been investigated.

Abbreviations: GD, gestational day; HPA, hypothalamo-pituitary-adrenal; TCA, thrichloroacetic acid; TNF- $\alpha$, tumor necrosis factor- $\alpha$ 


\section{METHODS}

Animals. Timed-mated Wistar rats (B\&K Universal, Sollentuna, Sweden) were maintained under controlled noise-free conditions ( $12 \mathrm{~h}$ light/ $12 \mathrm{~h}$ dark cycle, temperature $21 \pm 2^{\circ} \mathrm{C}$; humidity, 55-65\%) and fed standard rat pellets ad libitum. Standard principles of laboratory animal care were followed, and all procedures were approved by the Animal Ethics Committee of the University of Göteborg.

Isotopes. Unlabeled recombinant human IL-6 was purchased from Sigma Chemical Co. Aldrich (Sigma Chemical Co--Aldrich, St. Louis, MO, USA) and ${ }^{125} \mathrm{I} \mathrm{IL}-6$ (specific activity $100 \mu \mathrm{Ci} / \mu \mathrm{g}$ ) from PerkinElmer (PerkinElmer Life Science, Inc. Boston, USA). The radioactive half-life of ${ }^{125}$ I IL-6 is $60 \mathrm{~d}$ and the batches used were labeled the day before the experiment. The labeled interleukin was separated from free iodine $(<5 \%)$ and hydrated in $0.1 \mathrm{M}$ sodium phosphate, $5 \%$ sucrose, $0.25 \%$ BSA and a stabilizer at $\mathrm{pH} 6.8$. Incorporation of ${ }^{125} \mathrm{I}$, as determined by acid precipitation, was $>95 \%$ and the activity was $40.7 \mu \mathrm{Ci} / \mathrm{mL}(70.000 \mathrm{cpm} / \mu \mathrm{L}) .{ }^{14} \mathrm{C}$ methyl-glucose $(100 \mu \mathrm{Ci} / \mathrm{mL}$, in saline) was purchased from NEN Life Sciences Products (Boston, MA). Sodium iodide was obtained from Sigma Chemical Co. Aldrich.

Protocol. Dams (weight range 225-260) were anesthetized with 125 $\mathrm{mg} / \mathrm{kg}$ body weight thiobutabarbital sodium (Inactin, RBI, Natick, MA) at gestation day (GD) 11-13 $(n=5)$ or at GD 17-19 $(n=6)$. Catheters were inserted into the left carotid artery for blood sampling and into the right jugular vein for infusions. In preliminary studies, $10 \mu \mathrm{g} / \mathrm{kg}$ of NaI was administered ip 30 min before infusion of ${ }^{125}$ I IL-6. The administered dose of unlabeled sodium iodide was calculated to result in approximately 1,000-fold higher concentration of unlabeled iodide than labeled iodide even if all ${ }^{125} \mathrm{I}$ were released from ${ }^{125}$ I-IL-6. Thus, under these experimental conditions, transplacental transport of free ${ }^{125} \mathrm{I}$ is greatly reduced by competition with non radioactive iodine.

${ }^{125} \mathrm{I}$ IL-6 $(1.4 \mathrm{~mL}(57 \mu \mathrm{Ci}) / \mathrm{kg}$ body weight $)$ was given as an infusion $(0.1 \mathrm{~mL} / \mathrm{min})$ and maternal blood samples (each sample $200 \mu \mathrm{L}$ ) were collected from dams before and 1, 3, 5, 8, 10, 12, 15, 20, 25 and 30 min after the infusion. Subsequently, i.e., $30 \mathrm{~min}$ after the start of the infusion, the procedure to harvest the fetuses started. A lower midline abdominal incision was performed and the uterus was opened. Amniotic fluid was collected using a sterile needle $(1.20 \times 50 \mathrm{~mm})$, the amniotic sac was opened and the fetus and placenta was gently extracted, carefully avoiding blood contamination. Subsequently, the dam was killed with decapitation and the right maternal kidney was rinsed in saline to remove excess blood. Amniotic fluid, fetuses, placentas as well as maternal right kidney were weighed, and radioactivity was measured in a gamma-counter (Perkin-Elmer). All samples had counts well above $100 \mathrm{cpm}$, the level below which the supplier cannot guarantee reliable counting.

In a separate series of control experiments, three dams received ${ }^{14} \mathrm{C}$ methyl-glucose ( $2 \mu \mathrm{Ci} / 100 \mathrm{~g}$ body weight) iv at day 19 of pregnancy using the same protocol as for the ${ }^{125}$ I IL-6 experiments. Placentas, fetuses and maternal kidney were homogenized in distilled water (1:3 wt/vol), 20\% thrichloroacetic acid (TCA) added and homogenates were centrifuged at $10,000 \mathrm{~g}$ for $10 \mathrm{~min}$ and supernatant (S1) and pellets collected. The pellet was washed with 5-10 $\mathrm{mL}$ TCA $(5 \%)$, centrifuged at $10,000 \mathrm{~g}$ for $10 \mathrm{~min}$, and the supernatant (S2) collected. The combined supernatants (S1 and S2) were counted in a liquid scintillation counter.

Data analysis. Data are presented as mean \pm SEM. Since observations in individual fetuses and placentas of the same litter are not independent data, an average was obtained for each litter. The amount of radioactivity recovered from $1 \mathrm{~g}$ of organ or fetus was related to the cpm in $1 \mathrm{~mL}$ of maternal blood by the formula R-value $=(\mathrm{cpm} / \mathrm{g}$ organ $) /($ average $\mathrm{cpm} / \mathrm{mL}$ maternal blood). With a $\mathrm{R}$-value at 1 the isotope has equilibrated across the barrier indicating that fluxes are similar in the two directions across the placental barrier, whereas if $\mathrm{R}<1$ then the radioactive concentration is higher in the maternal circulation compared with the fetal side. Unidirectional maternofetal clearence was calculated using the formula $\mathrm{K}_{\mathrm{mf}}=$ total $\mathrm{cpm}$ in fetus/(placental weight in gram multiplied with area under the curve of the radioactivity from maternal blood $(0-30 \mathrm{~min})$ ).

Differences between the mid-gestation group (GD 11-13) and late gestation group (GD 17-19) were evaluated statistically using MannWhitney $U$-test. Paired comparisons between tissues were performed with Wilcoxon rank sum test. A $p$-value $<0.05$ was considered statistically significant.

\section{RESULTS}

There was no difference in mean litter size between the mid-gestation group (9.6, range 6-12) and the late gestation group $(9.5$, range $8-10)$. After infusion of ${ }^{125}$ IL-6, the levels in maternal blood reached a peak level at $1 \mathrm{~min}$ and a plateau after $10 \mathrm{~min}$. There was no significant difference in the levels of radioactivity achieved in maternal blood between the two experimental groups (Fig. 1).

The experiments in which a high dose of unlabeled $\mathrm{NaI}$ was administered before infusion of ${ }^{125} \mathrm{I}$ IL- 6 allowed for assessment of the contribution of free-labeled iodide to the radioactivity recovered from the fetus. Administration of unlabeled iodine did not significantly affect the counts measured in the fetus (data not shown). This finding led us to assume that ${ }^{125} \mathrm{I}$ which had dissociated completely from IL-6 did not contribute to fetal radioactivity.

The R-values calculated for fetus, amniotic fluid and maternal kidney are shown in Table 1 . The radioactivity recovered per gram fetus in late gestation was $0.4 \%$ of the radioactivity in $1 \mathrm{~mL}$ of maternal blood (R-value 0.004), suggesting only limited passage of IL- 6 across the placental barrier at this stage of pregnancy. In marked contrast, the R-value for IL-6 was 40 -fold higher in mid-pregnancy $(p<0.01)$. This difference was mirrored in the much higher unidirectional maternofetal clearance for IL-6 in mid-pregnancy compared with late pregnancy (Table 2). The R-value for IL-6 in maternal kidney was similar in both experimental groups and above one, as expected (Table 1). The high isotope content in maternal kidneys is likely to be due to multiple mechanisms. First, the kidney has a very high blood flow per $\mathrm{g}$ tissue and ${ }^{125}$ I-IL-6 in circulating blood in the kidney is likely to contribute substantially to the total counts. Second, specific binding of IL-6 to its receptor (IL-6Ra/gp130 complex), abundantly expressed in the kidney in particular in mesangial cell (22), may contribute. Third, the kidney represents a primary route of clearance for IL-6 and we suggest that renal breakdown ${ }^{125}$ I-IL-6 will result in the accumulation of ${ }^{125} \mathrm{I}$-IL-6 fragments and free ${ }^{125} \mathrm{I}$ in renal tissue and primary urine.

The fetal R-value for methyl-glucose was found to be 1 (Table 1). This is in agreement with methyl-glucose being a molecule that rapidly is distributed in the extra and intracellular volume and is efficiently transported by the placental glucose transporters. Furthermore, the unilateral materno-fetal

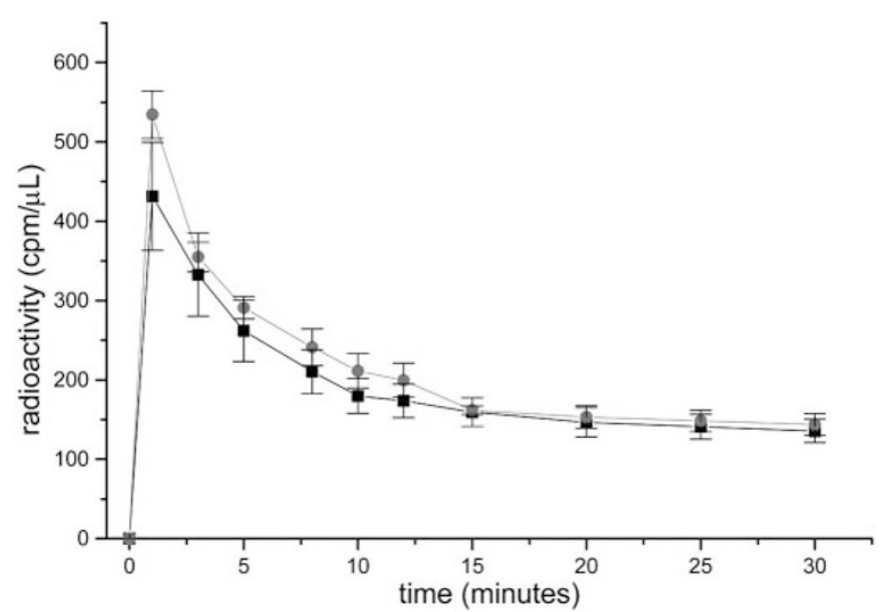

Figure 1. The concentration of radioactivity in the blood of the dams in mid $(\mathbf{O} ; n=5)$ and late pregnancy $(\square ; n=6)$ from $0-30 \mathrm{~min}$ after i.v. administration of ${ }^{125}$ I IL-6. The values are given as mean \pm SEM. 
Table 1. The R-values for ${ }^{125} I I L-6$ and ${ }^{14} C$-Methyl-glucose in mid (GD 11-13) and late gestation (GD 17-19)

\begin{tabular}{lccc}
\hline \multicolumn{1}{c}{ Sample } & $\begin{array}{c}{ }^{125} \mathrm{IL}-6 \\
\text { Mid-gestation } \\
(\mathrm{n}=5)\end{array}$ & $\begin{array}{c}{ }^{125} \mathrm{IL}-6 \\
\text { Late gestation } \\
(\mathrm{n}=6)\end{array}$ & $\begin{array}{c}{ }^{14} \mathrm{C}-\text { Methyl-glucose } \\
\text { Late gestation } \\
(\mathrm{n}=3)\end{array}$ \\
\hline Fetus & $0.17 \pm 0.03$ & $0.004 \pm 0.01 *$ & $1.02 \pm 0.10$ \\
Amnion fluid & - & $0.011 \pm 0.01$ & $0.23 \pm 0.02$ \\
Maternal kidney & $1.32 \pm 0.32$ & $1.42 \pm 0.29$ & $2.01 \pm 0.21$ \\
\hline
\end{tabular}

The values are given as mean \pm SEM.

$\mathrm{R}$-value $=$ (c.p.m./g organ) $/($ average c.p.m./mL maternal blood $)$.

$* p<0.01$ comparing mid versus late gestation with Mann-Whitney U-test.

Table 2. The unidirectional maternofetal clearance in mid (GD 11-13) and late gestation (GD 17-19)

\begin{tabular}{cccc}
\hline & $\begin{array}{c}{ }^{125} \mathrm{IL}-6 \\
(n=5)\end{array}$ & $\begin{array}{c}{ }^{125} \mathrm{IL}-6 \\
(n=6)\end{array}$ & $\begin{array}{c}{ }^{14} \mathrm{C}-\text { Methyl-glucose } \\
\text { Late gestation } \\
(n=3)\end{array}$ \\
\hline $\mathrm{K}_{\mathrm{mf}}(\mu \mathrm{l} / \mathrm{min})$ & $5.23 \pm 1.047$ & $0.34 \pm 0.059^{*}$ & $77.10 \pm 8.581$ \\
\hline
\end{tabular}

The values are given as mean \pm SEM.

$\mathrm{K}_{\mathrm{mf}}=$ c.p.m. in fetal tissue/(placenta weight (g)*AUC blood).

$* p<0.01$, comparing mid versus late with Mann-Whitney U-test.

clearance for methyl-glucose was $>200$-fold higher than for IL-6 (Table 2), reflecting the major difference in molecular size and the presence of specific transport systems for methylglucose in the placental barrier.

\section{DISCUSSION}

In this study we show that IL-6 administered to pregnant rats in mid or late pregnancy is transferred across the placental barrier, allowing direct exposure of the fetus. However, there was a striking difference between the two gestational stages studied with markedly higher IL-6 transfer at GD 11-13 compared with GD 17-19.

There are several methodological aspects of this study that needs to be considered. To obtain an accurate estimate of unidirectional clearances with the technique used in the current study it is important that the degree of back-flux of isotope in the fetal-maternal direction is low. We estimated the back-flux for IL-6 in the late pregnant group to be close to zero as indicated by the R-value $(0.4 \%)$. In the mid-pregnant group the R-value (17\%) indicates that back-flux was starting to become significant at the end of the experimental period. This will however only cause a relatively small underestimation of calculated unidirectional clearance.

A further concern is whether the recovered counts from the fetus represent intact, and therefore bioactive, IL-6 or whether there is a contribution of free ${ }^{125} \mathrm{I}$ or isotope labeled breakdown fragments of ${ }^{125} \mathrm{I}$-IL-6. This is particularly important for the mid-pregnancy group, which had a markedly higher measured unidirectional clearance of ${ }^{125}$ I-IL-6. In a preliminary series of experiments unlabeled iodide was administered in a dose resulting in concentrations 1,000-fold higher than would result if all iodide in ${ }^{125}$ I-IL-6 were released. As a consequence, mediated transplacental transfer of ${ }^{125} \mathrm{I}$ is completely inhibited under these experimental conditions. These experiments demonstrated that free ${ }^{125} \mathrm{I}$ did not contribute to fetal counts. However, the possibility that isotope-labeled fragments of IL-6 (from the maternal compartment or as a result of placental metabolism) contribute to fetal counts remains. This possibility could, for example, be addressed experimentally by assessing to which extent fetal counts represented intact IL-6 using HPLC or ELISA. However, the small size of the rat fetus in mid-pregnancy precludes the collection of plasma samples making application of these techniques difficult. Therefore we cannot exclude that there is a contribution of breakdown products of ${ }^{125}$ I-IL-6 to fetal counts, representing a possible limitation of this study.

Large molecules such as IL-6 (approximately $30 \mathrm{kDalton}$ ) may cross the placenta by simple diffusion through large transtrophoblastic water-filled channels or transfer may be mediated by a specific transport system. The physiologic evidence for the existence of transtrophoblastic channels is compelling (23) whereas morphologic evidence for these pathways has been more elusive (24). Specific placental transport systems for large peptides have been described, the most well-known example being the transfer of maternal immunoglobulins mediated by binding to the $\mathrm{Fc}$ receptor on the maternal surface of the placenta (25). Another example is leptin, transported across the rat placenta after binding to the C-terminally truncated isoform of the leptin-receptor (26).

The permeability of the placenta increases with increasing gestation, so during the last third of gestation the passive permeability to nonelectrolyte solutes of the rat placenta will increase markedly (27). Whereas the unidirectional maternofetal clearance $\left(\mathrm{K}_{\mathrm{mf}}\right)$ for mannitol ( $\left.\mathrm{mw} 180\right)$ increased 7-fold from GD 15 to GD 22, $\mathrm{K}_{\mathrm{mf}}$ for inulin (mw 5000) increased 3.5 -fold during the same gestational period (27). The most likely explanation for these data are that transtrophoblastic water-filled channels ("pores") become more numerous and narrower toward end of gestation. Thus, the diffusion of large tracers such as inulin becomes restricted in late gestation. Although studying different tracers it is of interest to compare $\mathrm{K}_{\mathrm{mf}}$ values in our late gestation group with values for GD 18 in the study of Atkinson and coworkers (27). The $\mathrm{K}_{\mathrm{mf}}$ value reported for inulin was 2-fold higher than the $\mathrm{K}_{\mathrm{mf}}$ value for IL-6 in the present study, which is in line with the larger molecular size of IL-6. Furthermore, the $\mathrm{K}_{\mathrm{mf}}$ value for methylglucose in our series of experiments was 15 -fold higher than that reported for mannitol (27), which is of the same molecular size as methyl-glucose. This difference illustrates increased rate of transfer above simple diffusion that is due to placental glucose transporters.

Considering the substantial increase in passive permeability of the rat placenta from GD 16 to 22 (27), the markedly higher rate of transplacental IL-6 transfer at GD 11-13 compared with late gestation was unexpected. Indeed, the $\mathrm{K}_{\mathrm{mf}}$ for IL-6 in mid-gestation in our study was of the same magnitude as $\mathrm{K}_{\mathrm{mf}}$ values reported for mannitol in late gestation (27). There are several possible explanations to these findings. First, due to the small fetal and placental size at GD 11-13 careful dissection techniques are critical to avoid any contamination of maternal blood. Since careful procedures were used it is unlikely that contamination of fetal tissue with isotope in maternal blood significantly have contributed to these results. Second, the higher transfer of IL-6 found at GD 11-13 compared with late pregnancy (GD 17-19) might be explained 
by the inverted yolk sac placenta of the rat which is the major source of maternal/fetal exchange from GD 7 to GD 12 and thereafter supplanted by the chorioallantoic placenta which is the only placenta in humans (28). However, if the rat yolk sac placenta is markedly more permeable to large molecules than its chorioallantoic counterpart it would be expected that placental transfer of IL- 6 would be inversely related to gestational age between GD 11 (primarily yolk sac placenta) and GD 13 (primarily chorioallantoic placenta). Although the number of experiments was limited in our mid-gestation group, no such association could be observed. Furthermore, the high transplacental IL-6 transfer in mid- compared with late gestation may be related to the presence of significantly wider "paracellular" water-filled channels at this stage of pregnancy.

Another possibility is that transplacental transfer of IL-6 is mediated by specific mechanisms. IL-6 binds to the IL-6Ra/ gp130 receptor complex and it has been demonstrated that it is the gp130 subunit that is important for internalisation of IL-6 bound to its receptor (29). Therefore a higher expression of gp130 in the maternal-fetal interface in mid-gestation than at the end of pregnancy might provide a basis the higher IL-6 permeability in mid-gestation observed in the present. Alternatively, specific placental transport systems for IL-6 may be present at mid-gestation which become down-regulated later in pregnancy. Moreover, if there are gestational age differences in placental IL- 6 permeability then there could also be gestational age differences in fetal membrane IL- 6 permeability. These possibilities need to be addressed in further studies including blocking transport with high levels of unlabeled IL-6 and testing tracers of different sizes.

Our data suggest that IL-6 is readily transferred to the fetus in mid-gestation whereas transfer is more limited in late gestation. However, this does not necessarily mean that the fetus is efficiently protected against maternal IL- 6 at the end of pregnancy since a significant amount of this cytokine will cross the placental barrier if maternal levels are sufficiently high. We have previously shown that the dose of IL-6 administered in the present study cause hypertension, insulin resistance, elevated stress response and dysregulation of the hypothalamic-pituitary-adrenal axis activity in the offspring in adult age $(11,12)$. Although any extrapolation to the human must be carried out with caution, the general permeability characteristics of the rat and human placenta are similar despite the difference in structure (21). Furthermore, this dose results in maternal IL-6 plasma concentrations similar to those observed in fulminant infection (1). Circulating IL-6 levels in adult patients with community-acquired pneumonia on admission have a mean of $477 \mathrm{pg} / \mathrm{mL}$ (1). Using our $\mathrm{R}$-value for IL-6 in the late pregnant rat in the present study (0.004), mean fetal concentrations would be expected to be approximately $2 \mathrm{pg} / \mathrm{mL}$. However, fetal plasma concentrations of IL-6 are likely to be 3-5 times higher since IL- 6 will not be distributed intracellular. Interestingly, IL-6 levels measured in umbilical vein during maternal chorioamnionitis can reach $25 \mathrm{pg} / \mathrm{mL}$ (30). Perinatal asphyxia is a common cause of neonatal morbidity, mortality and of long-term disabilities among survivors (31). In a recent study, the degree of encephalopathy and long-term outcome in term infants with perinatal asphyxia, IL-6 levels in serum and cerebrospinal fluid with cut-off values of 9.5 and $25.9 \mathrm{pg} / \mathrm{mL}$, respectively, were highly predictive for adverse outcome at $2 \mathrm{y}$ of age (32). Thus, even with limited placental transfer of IL-6 in late pregnancy it is possible that maternally derived cytokines may result in fetal plasma concentrations that may be harmful to the fetus.

Recently Zaretsky and coworkers reported that IL-6, but not IL-1a or TNF-a, was transferred across the in vitro perfused term human placenta (15). In contrast, the human term placenta appears not to be permeable to IL-8 (14). Selective permeability to IL-6 implicates a specific mediated pathway for transplacental transfer of IL-6. Since elevated fetal cytokine levels may represent a clinically important problem primarily in the late second and early third trimester, our data showing marked differences in placental IL-6 permeability with gestational age suggest that human placental IL-6 transfer need to be studied earlier in pregnancy. To conclude, in the pregnant rat studied in vivo, maternally administered IL-6 is transferred to the fetus both in mid and late gestation. However, the placental permeability to IL-6 is markedly higher at GD 11-13 than at GD 17-19. Further studies are warranted to address the question whether there is a mediated placental transport of interleukines and if this is true also in human pregnancies. If this is the case, it may have important implications, such as presenting a possibility to block this transfer pharmacologically.

Acknowledgments. The authors are grateful to S. Arnardottir for her laboratory work, and G. Ekeroth for statistical support.

\section{REFERENCES}

1. Antunes G, Evans SA, Lordan JL, Frew AJ 2002 Systemic cytokine levels in community-acquired pneumonia and their association with disease severity. Eur Respir J 20:990-995

2. Patrick MJ 1967 Influence of maternal renal infection on the foetus and infant. Arch Dis Child 42:208-213

3. Dammann O, Leviton A 1997 Maternal intrauterine infection, cytokines, and brain damage in the preterm newborn. Pediatr Res 42:1-8

4. Urakubo A, Jarskog LF, Lieberman JA, Gilmore JH 2001 Prenatal exposure to maternal infection alters cytokine expression in the placenta, amniotic fluid, and fetal brain. Schizophr Res 47:27-36

5. Romero R, Yoon BH, Kenney JS, Gomez R, Allison AC, Sehgal PB 1993 Amniotic fluid interleukin-6 determinations are of diagnostic and prognostic value in preterm labor. Am J Reprod Immunol 30:167-183

6. Yoon BH, Park CW, Chaiworapongsa T 2003 Intrauterine infection and the development of cerebral palsy. BJOG 110:124-127

7. Mittendorf R, Montag AG, MacMillan W, Janeczek S, Pryde PG, Besinger RE, Gianopoulos JG, Roizen N 2003 Components of the systemic fetal inflammatory response syndrome as predictors of impaired neurologic outcomes in children. Am J Obstet Gynecol 188:1438-1446

8. Barker DJ 1990 The fetal and infant origins of adult disease. BMJ 301:1111

9. McEwen BS 1997 Stress, brain, and behaviour: life-long effects upon health and disease. In: Kinney JM, Tucker HN (eds) Physiology, stress and malnutrition: functional correlates, nutritional intervention. Lippincott-Raven, Philadelphia. pp $113-130$

10. Weinstock M 2001 Alterations induced by gestational stress in brain morphology and behaviour of the offspring. Prog Neurobiol 65:427-451

11. Dahlgren J, Nilsson C, Jennische E, Ho HP, Eriksson E, Niklasson A, Bjorntorp P, Albertsson Wikland K, Holmang A 2001 Prenatal cytokine exposure results in obesity and gender-specific programming. Am J Physiol Endocrinol Metab 281:E326-E334

12. Samuelsson A-M, Öhrn I, Dahlgren J, Eriksson E, Angelin B, Folkow B, Holmäng A 2004 Prenatal exposure to IL-6 results in hypertension and increased hypothalamic-pituitary-adrenal axis activity in adult rats. Endocrinology 145:4897-4911

13. Chandorkar GA, Stobaugh JF, Audus KL 1999 Peptid transport and metabolism across the placenta. Adv Drug Deliv Rev 38:59-67

14. Reisenberger K, Egarter C, Vogl S, Sternberger B, Kiss H, Husslein P 1996 The 
transfer of interleukin- 8 across the human placenta perfused in vitro. Obstet Gynecol 87:613-616

15. Zaretsky MV, Alexander JM, Byrd W, Bawdon RE 2004 Transfer of inflammatory cytokines across the placenta. Obstet Gynecol 103:546-550

16. Abzug MJ, Rotbart HA, Magliato SA, Levin MJ 1991 Evolution of the placental barrier to fetal infection by murine enteroviruses. J Infect Dis 163:1336-1341

17. Ferro EA, Silva DA, Bevilacqua E, Mineo JR 2002 Effect of Toxoplasma gondii infection kinetics on trophoblast cell population in Calomys callosus, a model of congenital toxoplasmosis. Infect Immun 70:7089-7094

18. Kent AS, Sullivan MH, Elder MG 1994 Transfer of cytokines through human fetal membranes. J Reprod Fertil 100:81-84

19. Nilsson C, Larsson BM, Jennische E, Eriksson E, Bjorntorp P, York DA, Holmang A 2001 Maternal endotoxemia results in obesity and insulin resistance in adult male offspring. Endocrinology 142:2622-2630

20. Stulc J, Stulcova B 1993 Asymmetrical transfer of inert hydrophilic solutes across rat placenta. Am J Physiol 265:R670-R675

21. Robinson NR, Atkinson DE, Jones CJ, Sibley CP 1988 Permeability of the nearterm rat placenta to hydrophilic solutes. Placenta 9:361-372

22. Kammüller ME 1995 Recombinant human interleukin-6: safety issues of a pleitrpic growth factor. Toxicology 105:91-107

23. Stulc J 1989 Extracellular transport pathways in the hemochorial placenta. Placenta 10:113-119

24. Kertschanska S, Stulcova B, Kaufmann P, Stulc J 2000 Distensible transtrophoblastic cahennels in the rat placenta. Placenta 21:670-677
25. Simister NE 2003 Placental transport of immunoglobulin G. Vaccine 21:3365-3369 Review

26. Smith JT, Waddell BJ 2003 Leptin distribution and metabolism in the pregnant rat: Transplacental leptin passage increases in late gestation but is reduced by excess glucocorticoids. Endocrinology 144:3024-3030

27. Atkinson DE, Robinson NR, Sibley CP 1991 Development of passive permeability characteristics of rat placenta during the last third of gestation. Am J Physio 261:R1461-R1464

28. Carney EW, Scialli AR, Watson RE, DeSesso JM 2004 Mechanisms regulating toxicant disposition to the embryo during early pregnancy: an interspecies comparison. Birth Defects Res C Embryo Today 72:345-360

29. Dittrich E, Rose-John S, Gerhartz C, Mullberg J, Stoyan T, Yasukawa K, Heinrich PC, Graeve L 1994 Identification of a region within the cytoplasmic domain of the interleukin-6 (IL-6) signal transducer gp130 important for ligand-induced endocytosis of the IL-6 receptor. J Biol Chem 269:19014-19020

30. Smulian JC, Vintzileos AM, Lai YL, Santiago J, Shen-Schwarz S, Campbell WA 1999 Maternal chorioamnionitis and umbilical vein interleukin-6 levels for identifying early neonatal sepsis. J Matern Fetal Med 8:88-94

31. Thornberg E, Thiringer K, Odeback A, Milsom I 1995 Birth asphyxia: incidence, clinical course and outcome in a Swedish population. Acta Paediatr 84:927-932

32. Tekgul H, Yalaz M, Kutukculer N, Ozbek S, Kose T, Akisu M, Kultursay N, Gokben S 2004 Value of biochemical markers for outcome in term infants with asphyxia. Pediatr Neurol 31:326-332 